\title{
Mass gathering public health and emergency medicine literature review: Levels of evidence
}

\author{
Paul Arbon PhD, Lynette Cusack PhD, Naomi Verdonk B.Oenol(Hons) \\ Affiliation: \\ Flinders University Disaster Research Centre, GPO Box 2100, Adelaide, South Australia, Australia.
}

\section{ABSTRACT}

\section{Background}

The science of mass gatherings is a relatively new and developing field. It is currently at a stage where summarising the rigour of knowledge gained about the complex interrelationships between key characteristics of an event, spectator profiles and health implications are critical. This study seeks to summarise the levels of evidence in peer-reviewed journal articles concerning mass gathering public health and emergency medicine published 2001 to 2011. Until now, the evidence behind the science of mass gathering public health and emergency medicine has not been critically analysed.

\section{Methods}

Publications were reviewed by searching the following online databases: GALE, NLM, Web of Science, Elsevier, Wiley, BMJ Journals, OUP, IngentaConnect, RMIT, DOAJ and JSTOR. Published news articles and grey literature were omitted. The peer-reviewed articles were organised into pre-determined World Health Organisation categories and the levels of evidence were assessed using the effectiveness classifications developed by the Joanna Briggs Institute. Descriptive statistical analysis was then undertaken using Microsoft Excel®.

\section{Results}

Of all publications examined, $38 \cdot 86 \%(n=89)$ of the articles found in this review were categorised as observational studies, $36 \cdot 68 \%(n=84)$ were expert opinion or consensus, $20 \cdot 09 \%(n=46)$ were cohort studies, $2 \cdot 18 \%(n=5)$ were case-controlled studies and $2 \cdot 18 \%(n=5)$ were quasi-experimental studies.

\section{Conclusion}

High-level evidence studies may not be possible in the mass gathering context, but research in the middlelevel should be encouraged to ensure that literature is less reliant on experience and expert opinion when applied to event management strategies which impact on public health and emergency medicine.

\section{Keywords}

Mass gatherings; emergency medicine; public health; literature review; levels of evidence

\section{INTRODUCTION}

There have been a number of important literature reviews undertaken in the past related to specific aspects of mass gathering medicine. In 1997, De Lorenzo published a review which assessed the state of scientific knowledge at that time and challenged researchers to address key issues in the provision of an effective healthcare service during a mass gathering event.(1) In 2002, Milsten et al provided an extensive literature review using searches of Medline and Healthstar from 1997 to May 2002, to identify the variables associated with mass gatherings. In effect, he took stock of progress in this specific area of mass gatherings over the five-year period since the De Lorenzo review.(2) 
This paper revisits and reviews the multiple, interacting variables that add an element of uncertainty to planning for mass gatherings and argued that a better understanding of these variables could improve event management.(2) In 2004, Arbon noted the preponderance of descriptive papers in the literature and argued for more critical work including the development of theory and conceptual models to encourage greater levels of research in the field of massgathering medicine.(3) Arbon noted that a review of the literature on profile of casualty types and range suggests that mass gathering health research is characterized by an excess of isolated studies that are not linked to an integrated theoretical framework and subsequently there are deficiencies in the theoretical basis to support and guide practice.(3)

The science of mass gatherings is a relatively new and developing field. It is currently at a stage where summarising the rigour of knowledge gained about the complex interrelationships between key characteristics of an event, patient profiles and public health implications are critical. The purpose of this research is to undertake a review of online peer-reviewed scholarly articles concerning mass gathering public health and emergency medicine published between 2001 and 2011. This study organises the articles into predetermined World Health Organisation (WHO) categories, as well as measure the levels of evidence using the effectiveness classifications developed by the Joanna Briggs Institute (JBI). The WHO categories are those tentatively identified as section headings for the redevelopment of the WHO Guidelines for mass gathering health. This is useful to understanding the development of mass gathering literature because it provides information about the level of evidence in publications.

\section{METHODS}

Publications concerning public health and emergency medicine aspects of mass gatherings were reviewed. A key word search was conducted to locate scholarly peer-reviewed articles published in English between 2001 and 2011 by searching the following online databases; GALE, NLM, Web of Science, Elsevier, Wiley, BMJ Journals, OUP, IngentaConnect, RMIT, DOAJ and JSTOR. The extensive search was undertaken by combining the terms Emergenc* OR Public Health with Mass gathering*, Event*, Crowd*, World Cup, Rall* (rally, rallies, rallying etc), Soccer, Football, Superbowl, Rock, Concert*, Jamboree*, Hajj, Pope, Papal, Lourdes, Fatima, Pilgrim*, Festival*, Cricket, Marathon*, Rugby, Olympi* (Olympics, Olympiad etc), Game*, Tennis, Sport*, Demonstration*, Protest*, Summit, Riot*, Flash mob, Arena and Stadi* (stadia, stadium). Wild card entries were utilised to ensure that all variations of a term were included in the search. To qualify for inclusion, a publication had to be about public health or emergency medicine, irrespective of whether mass gatherings were studied generally or specifically. The search resulted in over 3000 articles which were refined to 721 by examining the title, abstract and key words to ensure that they were relevant to general mass gathering health. Full text publications were identified; however published news and commentary articles were omitted. Further to this, a review of the article's subject matter was undertaken by reading each paper to ensure that only mass gathering public health and emergency medicine topics were included. The final number of relevant articles was reduced to 229 which were classified as either relating to public health or emergency medicine. The data was processed and analysed in Microsoft Excel $\circledast$ using frequencies and tables as well as sums and counts.

The articles were grouped into their subject matter category, as determined by the WHO (Nunn M, Personal Communication, October 2011). The categories comprised of:

1. Epidemiology

2. Event Operations

3. Disease Surveillance and Outbreak Response

4. Point of Entry Health

5. Use of Modern Technologies in Mass Gathering Planning and Operations

6. Psychosocial Considerations

7. Public Information and Health Promotion

8. Emergency Medical Services

9. Disaster Preparedness and Contingency Planning

10. Environmental Considerations

In addition, each publication was classified using the JBI levels of evidence with regard to effectiveness (4) as follows:

1. Meta-analysis (with homogeneity) of experimental studies (e.g. RCT with concealed randomisation) OR One or more large experimental studies with narrow confidence intervals

2. One or more smaller RCTs with wider confidence intervals OR Quasi-experimental studies (without randomisation)

3. a) Cohort studies (a control group)

b) Case-controlled

c) Observational studies (without control group)

4. Expert opinion, or physiology bench research, or consensus.(4) 


\section{RESULTS}

The following figures provide an overview of the classifications and levels of evidence of the articles found in this review, commencing with the number of public health and emergency medicine publications.

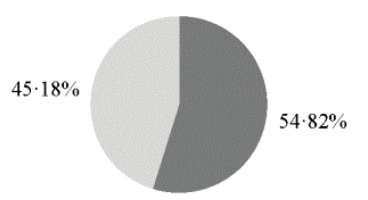

- Public Health $(\mathrm{n}=126) \quad$ Emergency Medicine $(\mathrm{n}=103)$

Figure 1: Subject matter of the articles found in this review $(n=$ 229)

Figure 2 shows the percentage of articles in each WHO category found in this review for both public health and emergency medicine.

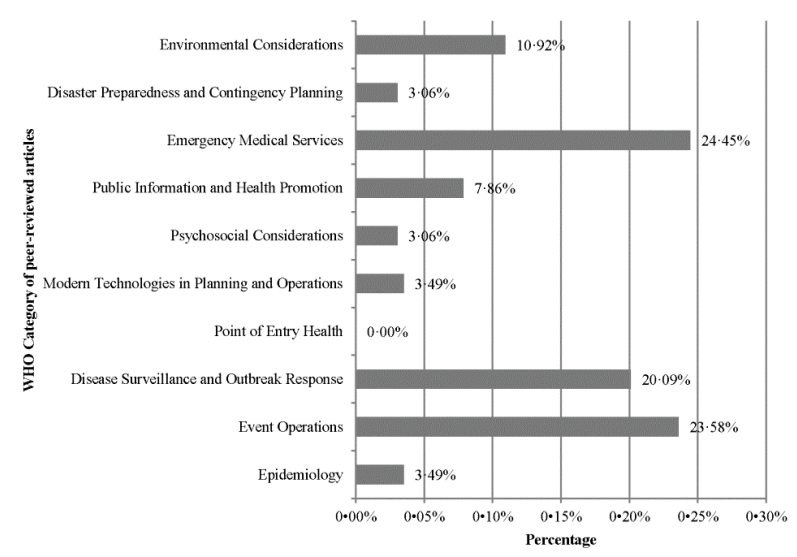

Figure 2: Percentage of articles in each WHO category found in this review.

Figure 3 indicates the JBI effectiveness levels of evidence of the articles organised into WHO categories found in this review.

The JBI effectiveness levels of evidence of the articles organised into WHO categories found in this review are summarised below:

\section{Epidemiology}

Of the journal articles classified into the category of Epidemiology $(n=8), 62.5 \%(n=5)$ concerned Pubic Health. All articles except two were graded to the third level of evidence. Of this group, $50 \cdot 00 \%(n=3)$ were cohort studies and the remainder were considered observational studies. The outstanding articles were classified as consensus studies and therefore met the criteria of the fourth category.

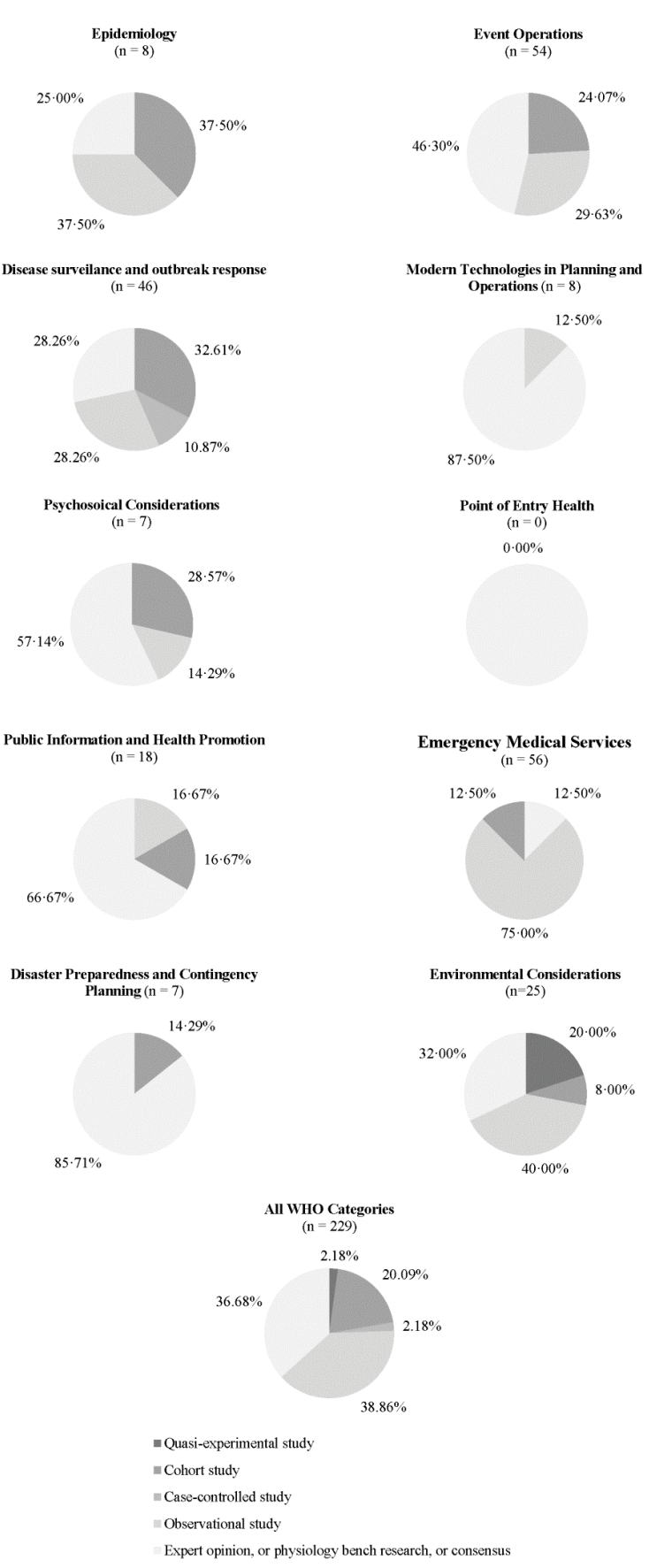

Figure 3: JBI effectiveness levels of evidence of the articles organised into WHO categories found in this review.

\section{Event Operations}

The subject of Event Operations $(n=54)$ is relevant to $23 \cdot 58 \%$ of the articles reviewed. $46.30 \%(n=25)$ of the articles met the criteria of the fourth JBI Category, $29.63 \%(n=16)$ were classified as observational studies and $24.07 \%$ (n $=13$ ) were cohort studies.

\section{Disease Surveillance and Outbreak Response Disease Surveillance and Outbreak Response concerned $20.09 \%(n=46)$ of all the articles reviewed. Most of the articles, $32 \cdot 61 \%(n=15)$ were cohort studies. $28 \cdot 26 \%(n=13)$ fell into the fourth category of the JBI levels of evidence ranking, another $28.26 \%(n=13)$ were}


observational studies and $10.87 \%(n=5)$ were case-controlled studies.

\section{The Use of Modern Technology}

The subject of the Use of Modern Technology related to $3 \cdot 49 \%(n=8)$ of all the articles reviewed. All articles except one $(n=7)$ were ranked in the fourth category of the JBI levels of evidence. The remaining article was an observational study.

\section{Point of Entry Health}

No peer-reviewed articles found in this review were categorised as Point of Entry Health.

\section{Psychosocial Considerations}

Psychosocial Considerations concerned 3.06\% (n $=7$ ) of all the articles reviewed. The majority of articles, $57 \cdot 14 \%(n=4)$ were either expert opinion or consensus, two articles were cohort studies and one was classified as an observational study. Public Information and Health Promotion The subject of Public Information and Health Promotion concerned $7 \cdot 86 \%(n=18)$ of all the articles reviewed. $66 \cdot 67 \%(n=12)$ were classified as an expert opinion or consensus, $16.67 \%(n=$ 3) were ranked as cohort studies and $16 \cdot 67 \%(n=$ 3) were observational studies.

\section{Emergency Medical Services}

Emergency Medical Services concerned $24 \cdot 45 \%$ $(n=56)$ of all the articles reviewed. $75 \%(n=42)$ of the articles were observational studies, $12.5 \%$ $(n=7)$ were cohort studies and $12 \cdot 5 \%(n=7)$ were either expert opinion or consensus.

\section{Disaster Preparedness and Contingency Planning \\ The subject of Disaster Preparedness and} Contingency Planning concerned $3.06 \%(n=7)$ of all the articles reviewed. $85 \cdot 71 \%(n=6)$ of these articles were classified as either an expert opinion or consensus, and one article was categorised as a cohort study.

\section{Environmental Consideration}

Environmental Considerations concerned 10.92\% $(n=25)$ of all the articles reviewed. $40 \%(n=10)$ of the articles were observational studies, $32 \%$ (n $=8$ ) were either expert opinion or consensus, $20 \%$ $(n=5)$ were quasi-experimental studies and $8 \%$ $(n=2)$ were cohort studies.

\section{All WHO categories}

Overall, $38 \cdot 86 \%(n=89)$ of the articles found in this review were categorised as observational studies, $36 \cdot 68 \%(n=84)$ were expert opinion or consensus, $20 \cdot 09 \%(n=46)$ were cohort studies, $2 \cdot 18 \%(n=5)$ were case-controlled studies and $2 \cdot 18 \%(n=5)$ were quasi-experimental studies.

\section{DISCUSSION}

The majority of peer-reviewed articles, 55.02\% $(n=126)$ found in this review concerned the public health implications of mass gathering events Figure 1). From 2001 to 2011 , articles related to Disease Surveillance and Outbreak Response were the most consistently represented in publications, followed by Emergency Medical Services and then Event Operations (Figure 2). Publications in each of the WHO categories are predominantly observational studies, then cohort studies, followed by expert opinion Figure 3 ). The highest JBI level of evidence measuring effectiveness in the mass gathering literature was level two; Quasi-experimental studies, mainly undertaken in the area of Environmental Considerations. There remains a strongly observational and descriptive approach to the science of mass gatherings with the levels of evidence at three and four. The findings at the lower levels of evidence limit the generalizability and veracity in their application to mass gathering management. In 2004, Arbon argued for more critical work including the development of theory and conceptual models to encourage research with higher levels of evidence in the field of mass gathering health.(3) Such progress was not evident from this review.

High-level evidence studies may not be possible in the mass gathering context, but studies in the middle-level should be encouraged so that there is less reliance on experience and expert opinion for event management strategies. There remains a need to strategically focus and further develop research in specific areas of mass gathering science to build a sound body of knowledge. As noted by Arbon back in 2004, mass gathering health research is characterised by an excess of isolated low level studies which are not linked to an integrated theoretical framework. This preponderance of lower evidence levels in the literature does not mean that these studies are uninformative or lack practical usefulness. The Holy Grail of mass gathering research may however remain the development of theoretical models supported by higher levels of evidence and capable of underpinning prevention and response strategies that are effective and suitable for application across different event types in varying contexts and locales. The development of theory will depend on the identification of the highest levels of evidence in each mass gathering health domain. A corollary to this requirement is the need for greater research effort especially in those fields where the amount and level of research is remarkably thin. There are deficiencies in the theoretical basis to support and guide mass gathering best practice.(3) This remains true today in 2012 . There is still a need to focus on developing research that can be applied 
and tested for the different mass gathering contexts.

\section{CONCLUSION}

There is a need for the development of theoretical models supported by higher levels of evidence to underpin the development of prevention and response models that are effective and suitable for application across the carious types and contexts of mass gatherings. Some aspects of mass gathering knowledge are very poorly developed and require considerable further work.

\section{CONFLICT OF INTEREST}

The authors declare they have no conflict of interest.

\section{REFERENCES}

1. De Lorenzo RA. Mass gathering medicine: a review. Prehosp Disaster Med. 1997; 12(1): 68-72.

2. Milsten AM, Maguire BJ, Bissell RA, Seaman KG. Mass-gathering medical care: a review of the literature. Prehosp Disaster Med. 2002; 17(3): 151-62.

3. Arbon P. The development of conceptual models for mass-gathering health. Prehosp Disaster Med. 2004; 19(3): 208-12.

4. Joanna Briggs Institute. Levels of Evidence FAME Systematic Reviews [cited 02 February 2012]; Available from: www.joannabriggs.edu.au/Levels $\% 20$ of $\% 20$ E vidence $\% 20 \% 20 \mathrm{FAME}$ 